\title{
Técnicas de programación neurolingüística ante la crisis Covid-19 una aproximación para el desarrollo de las habilidades productivas en la enseñanza del idioma Inglés en Ecuador
}

Neurolinguistic programming in the covid-19 crisis an approach for the development of productive skills in teaching English language in Ecuador

1 Diego Santiago Andrade Naranjo

Universidad de las Fuerzas Armadas ESPE, Departamento de Ciencias Humanas y

Sociales, Ambato, Ecuador

dsandrade3@espe.edu.ec

2 Carlos Enrique Proaño Rodríguez

Universidad de las Fuerzas Armadas ESPE, Departamento de Ciencias Humanas y

Sociales, Ambato, Ecuador

ceproanio@espe.edu.ec

3 Andrea Karina Carvajal Gavilanes

Universidad Técnica de Ambato, Facultad de Ciencias Humanas y de la Educación (E),

Ambato, Ecuador

acarvaja15121@uta.edu.ec

4 Narcisa de Jesús Mena Garzón

Universidad de las Fuerzas Armadas ESPE, Departamento de Ciencias Humanas y

Sociales, Ambato, Ecuador

ndmena@espe.edu.ec

Artículo de Investigación Científica y Tecnológica

Enviado: 24/12/2021

Revisado: 29/12/2021

Aceptado: 12/01/2022

Publicado:08/03/2023

DOI: https://doi.org/10.33262/concienciadigital.v6i1.4.2058

Cítese:

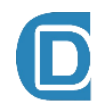

Ciencia Digital

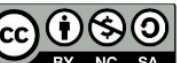

Andrade Naranjo, D. S., Proaño Rodríguez, C. E., Carvajal Gavilanes, A. K., \& Mena Garzón, N. de J. (2023). Técnicas de programación neurolingüística ante la crisis Covid-19 una aproximación para el desarrollo de las habilidades productivas en la enseñanza del idioma Inglés en Ecuador. ConcienciaDigital, 6(1.4), 1132-1145. https://doi.org/10.33262/concienciadigital.v6i1.4.2058

CONCIENCIA DIGITAL, es una Revista Multidisciplinar, Trimestral, que se publicará en soporte electrónico tiene como misión contribuir a la formación de profesionales competentes con visión humanística y crítica que sean capaces de exponer sus resultados investigativos y científicos en la misma medida que se promueva mediante su intervención cambios positivos en la sociedad. https://concienciadigital.org

La revista es editada por la Editorial Ciencia Digital (Editorial de prestigio registrada en la Cámara Ecuatoriana de Libro con No de Afiliación 663) www.celibro.org.ec

Esta revista está protegida bajo una licencia Creative Commons Attribution Non Commercial No

Derivatives 4.0 International. Copia de la licencia: http://creativecommons.org/licenses/by-nc-nd/4.0/ 
Palabras claves: neurolingüística, técnicas, habilidades productivas, Covid-19

Keywords: programming, neurolinguistics, techniques,

\section{Resumen}

La educación ha sufrido cambios importantes, a un más desde finales del año 2019 donde inicia una pandemia mundial que sin duda afecta la enseñanza y el aprendizaje en el idioma inglés en el Ecuador, el objetivo primordial de esta investigación fue determinar la influencia de las técnicas de programación neurolinguística en el desarrollo de las habilidades productivas en tiempos de pandemia, dado que los estudiantes no pueden expresarse correctamente de forma oral y escrita en sus clases virtuales, utilizando frases incorrectas, con poco uso de vocabulario y sin comprensión, lo que muestra baja autoestima e interés al expresarse en idioma inglés. La vergüenza y el miedo de cometer errores sin tener un entendimiento por parte del profesor y los compañeros de clase son otros aspectos importantes al momento de emitir un comentario o entablar un diálogo. En este contexto, se propuso realizar la presente experimentación con 30 estudiantes. Para este estudio se adoptó un diseño de grupo único pre y post-prueba utilizando una metodología de carácter cuantitativo realizado durante la pandemia. Los problemas de los estudiantes en el desarrollo de habilidades productivas fueron evaluados mediante el programa estadístico SPSS, aplicando el test estadístico T Student de pruebas relacionadas. Con base en los resultados de la preprueba, se aplicaron técnicas neurolingüísticas (Anclaje, Crear relación, Espejo, Mantener el flujo) durante 4 semanas en las clases presenciales y virtuales de inglés por la pandemia Covid-19. Al final de la experimentación, se realizó una post prueba, y el resultado del estudio reveló que los estudiantes a través de la programación neurolingüística, presentaron mejoras significativas en las habilidades productivas del idioma inglés, destacando que el uso de las estrategias mencionadas ayuda positivamente a la adquisición de un segundo idioma, especialmente en la forma de expresarse en inglés, dándoles mayor libertad y confianza a la hora de emitir un comentario oral y escrito en un segundo idioma.

\section{Abstract}

Education has undergone important changes, even more since the end of 2019 when a global pandemic begins that undoubtedly affects the teaching and learning of the English language in 
productive skills, Covid-19
Ecuador, the primary objective of this research was to determine the influence of neurolinguistics programming on development of Productive skills in times of pandemic, given that students cannot express themselves correctly orally and in writing in their usual virtual classes today, using incorrect phrases with little use of vocabulary and without understanding, which shows low selfesteem and interest in learning English. Shame and fear of making a mistake without understanding on the part of the teacher and classmates are other important aspects when making a comment or starting a dialogue. In this context, it was proposed to carry out the present experimentation with 30 students. For this study, a single group design was adopted before and after the test using a quantitative methodology carried out in the era of the pandemic. The problems of the students in the development of productive skills were evaluated using the SPSS statistical program, applying the Student's T or Statistical related tests. Based on the results of the pre-test, neurolinguistic strategies (anchoring, creating a relationship, mirroring, maintaining flow) were applied for 4 weeks in face-to-face and virtual English classes due to the covid 19 pandemic. At the end of the experimentation, a post-test, and the results of the study revealed that the students, through neurolinguistic programming, presented significant improvements in the productive skills of the English language, highlighting that the use of the aforementioned strategies positively helps the Acquisition of a second language, especially in the way they express themselves in English, giving them greater freedom and confidence when issuing an oral and written comment in a second language

\section{Introducción}

La declaración de pandemia por parte de la Organización Mundial de la Salud por COVID 19 ocasionó en el mundo entero un notable deterioro de la calidad de vida, afectando a familias y a la sociedad entera. Uno de los aspectos que también fue afectado es sin duda el ámbito educativo, transformándose en una enseñanza aprendizaje tradicional, de forma presencial, a un proceso virtual que con lleva un aprendizaje a través de medios informáticos. 
Las disrupciones provocadas por la COVID-19 en los estudiantes del Ecuador han ocasionado la perdida al acceso de un entorno estimulante y enriquecedor, oportunidades de aprendizaje, interacción social, y en algunos casos, nutrición adecuada, puesto que en varias instituciones públicas se fomentó la alimentación escolar como parte del proceso. Es probable que esta situación ponga en peligro su desarrollo saludable a largo plazo, especialmente en el caso de niños y adolescentes de familias de escasos recursos económicos; en este escenario de crisis pandémica, en la actualidad en el país se recibe clases en forma virtual, Con este antecedente y contexto se busca establecer un vínculo entre los principios de la Programación Neurolingüística --PNL-- (actividades destinadas a mejorar el estado emocional general de los estudiantes con el fin de facilitar el aprendizaje) pese a la crisis por COVID 19 y lograr una aproximación al desarrollo de las habilidad productivas (el acto de transmitir información de forma oral y escrita) en los estudiantes.

Al estudiar un idioma extranjero se deben considerar aspectos de desarrollo de habilidades comunicativas de manera efectiva. La comunicación efectiva es un tipo de comunicación en la que conseguimos transmitir el mensaje de una forma entendible y muy clara para el receptor sin provocar dudas, confusiones o posibles interpretaciones equivocadas (Castro, 2020).

Para Vargas (2021) el lenguaje como competencia comunicativa no es algo mecánico, sino que digiere, asimila, modela y elabora una expresión muy particular que se va dando en base a la actuación y relación con el mundo de manera activa, pues la experiencia abre el camino no continuar su proceso de desarrollo y madurez (p. 33).

Vargas (2021) expresa que la competencia linguística se sitúa en el acto del habla, en la realización, en el momento en que el hablante concreto utiliza el lenguaje como un medio para conseguir diversos objetivos en la locución como actividad.

Todas estas concepciones sobre el desarrollo del lenguaje desde habilidades o competencias lingüísticas conducen a enfocar la presente investigación desde un enfoque neurolingüística.

En concordancia del lenguaje y las habilidades se presenta un interés por este tema ya que se dio inicio al notar el bajo rendimiento académico de los estudiantes en especial por no encontrarse frecuentemente en clase presenciales. Su bajo rendimiento académico a lo largo del desarrollo escolar, sobre todo en la interacción al comunicarse con sus compañeros, docentes, evidencian la dificultad propia de estas habilidades y del idioma, que se puede medir por las rúbricas aplicadas en cada actividad. El mejorar la interacción oral y escrita mediante la programación neurolingüística ayudará en gran medida a los estudiantes a obtener mejores resultados, al mismo tiempo comunicarse con los demás en un segundo idioma. También es importante desarrollar esta investigación, ya que 
permitirá poner las técnicas de enseñanza actuales al servicio de los estudiantes para trabajar en sus problemas de aprendizaje del inglés. Estas cuestiones se han convertido en un problema social especialmente por el confinamiento, ya que al interferir en el desarrollo de la confianza en sí mismo no se abre el portal educacional para mejorar el desarrollo educativo de un país, y, por consiguiente, se limita también el logro del progreso económico.

Uno de los problemas más comunes de la educación de nivel medio es su calidad, que presenta deficiencias cuando no se actualiza y se adapta a las necesidades modernas de los estudiantes. Además, el estado de ánimo por estar en confinamiento por la pandemia hace que se generen constantes cambios, por lo cual es un tema donde se puede revelar el impacto de la investigación propuesta y muestra la eficacia con que las técnicas de programación neurolingüística marca el desarrollo emocional e intelectual de los estudiantes para generar habilidades productivas. Con la aplicación de estas técnicas, los padres, los estudiantes, los maestros y las autoridades se beneficiarán de la consecución del objetivo de mejorar el rendimiento y la calidad de la educación. Todo ello se hará con el objetivo de ayudar a los alumnos a dominar las habilidades, actitudes y aptitudes relacionadas con el uso del idioma inglés. Por último, los profesores tendrán la oportunidad de hacer frente a un nuevo reto consistente en utilizar técnicas y métodos no tradicionales para lograr el desarrollo del pensamiento crítico y el aprendizaje significativo en los estudiantes en tiempos de pandemia COVID 19.

\section{Covid-19 y su impacto en la educación}

El COVID 19 en el área educativa Trujillo-Sáez et al. (2020), manifiesta que los diferentes ámbitos como el; médico, químico, biológico y farmacológico el crecimiento de proyectos de investigación y publicaciones ha sido muy elevado, otros ámbitos como el educativo avanzan de manera más lenta, a pesar de ser una de las mayores preocupaciones sociales en todo el mundo, tanto durante la fase de confinamiento como en las posteriores fases de transición hacia la normalidad. Así, el número de publicaciones que relacionan la Educación con la pandemia de COVID-19 en bases de datos de revistas científicas de prestigio es francamente menor al número de publicaciones en otros ámbitos y éstas han quedado relegadas a la mínima expresión en plataformas de pre-prints internacionales (pp. 9-10). En tal virtud, es imperioso describir los hallazgos realizados sobre el mejoramiento del desarrollo de habilidades productivas en la comunicación en idioma inglés al haber aplicado técnicas de Programación Neurolinguística a un grupo de discentes, durante esta dura etapa de confinamiento y de educación en línea. 


\section{Habilidades de la comunicación en inglés}

Las habilidades de la comunicación en inglés están definidas en receptivas y productivas: las receptivas se componen de Listening y Reading, y las productivas por Writing y Speaking (Proaño et al., 2017).

Listening es la habilidad de entender mensajes verbales. The role of listening comprehension, dice que la comprensión auditiva precede la producción en todos los casos del aprendizaje de un idioma, y no puede haber producción a menos que se dé el estímulo lingüístico (linguistic input) y, que éste, a su vez, se convierta en material comprensible (comprehensible intake) para la persona que escucha. La comprensión es importante no sólo porque precede la producción lógica y cronológicamente, sino especialmente porque parece ser el mecanismo básico por medio del cual se internalizan las reglas del lenguaje.

Reading es la habilidad de comprender mensajes escritos. Las investigaciones revelan que tanto los conceptos de los docentes sobre lo que es aprender a leer como las actividades que se llevan a cabo en las aulas, no incluyen aspectos relacionados con la comprensión lectora. Esto pone de manifiesto que los docentes comparten mayoritariamente la visión de la lectura que corresponde a los modelos de procesamiento ascendente según los cuales la comprensión va asociada a la correcta oralización del texto.

Writing es la habilidad de producir documentos escritos. "La comunicación escrita es un modo de transmisión en el que el emisor elabora su mensaje sin necesidad de coincidir espacial y temporalmente con su receptor al momento de hacerlo. De hecho, ni siquiera es necesaria esa coincidencia efectiva. El sujeto propio de esta comunicación es el ser humano.

Speaking es la habilidad de expresar verbalmente un mensaje. "La comunicación oral es una de las dos formas tradicionales de expresión con que cuenta el ser humano y se distingue de la comunicación escrita en varios elementos fundamentales, como la presencia de los interlocutores involucrados o la duración del acto comunicativo en el tiempo (Proaño et al., 2017).

\section{Programación Neurolingüística}

La Programación Neurolingüística (PNL), se fundamenta en las concepciones y puntos de vista que asumen diferentes ciencias, sobre todo aquellas relacionadas con el desarrollo cognitivo y el lenguaje. Sus raíces se encuentran en la Linguiística, la Terapia de la Gestalt, la Semántica General, el Análisis transaccional, el Dominio Corporal, Cognitivo y Emocional (Estupiñan-Ricardo, 2017). 
La programación neurolingüística (PNL) a decir de Arlinthon \& Pomares (2011) fue creado por Jonh Grinder y Richard Bandler, a principios de los años de 1970. Bandler afirma al respecto que la programación Neuro-Lingüística es el nombre que inventó para evitar la especialización de un campo o en otro... una de las maneras que la (PNL) representa, es enfocar el aprendizaje humano; básicamente desarrollamos maneras de enseñarle a la gente a usar su propia cabeza (Bandler \& Gindler, 1980).

La programación neurolingüística se refiere al trabajo integrado entre el sistema neurológico y el lenguaje, las que en su articulación conforman aquellas estructuras que erigen los modelos del mundo circundante (Estupiñan-Ricardo, 2017).

\section{Técnicas de programación neurolingüística}

Los métodos de enseñanza de la Programación Neurolingüística se basan en la comunicación cuyo objetivo principal es crear una condición que motive a los estudiantes a aprender. Algunas técnicas que se describen a continuación nos permiten comprender cómo la PNL ayuda en el proceso de enseñanza-aprendizaje del idioma inglés. Según Yameen \& Iftikhar (2014) existen algunas técnicas que ayudan a los estudiantes a adquirir y aprender un segundo idioma de forma fácil:

El anclaje es una técnica de programación neurolingüística, esta es útil para regenerar una emoción llena de recursos. Trabaja en recrear la emoción mientras la asocia con la acción física. El anclaje crea una asociación con la emoción y la acción física elegida.

De esta manera la simpatía está asociada con la compenetración, la cual es la sensación de tranquilidad que se desarrolla cuando las personas interactúan con otras personas con las que se sienten cómodas y es esencial para que se produzca una comunicación significativa.

La simpatía es más probable cuando interactúan personas de ideas afines. En el aula, las actividades de convivencia y de 'llegar a conocerte', así como la negociación continua entre profesor y alumnos fomentan la compenetración, mientras que las actividades de brecha de comunicación y el trabajo en grupo la refuerzan.

Una forma de establecer una buena relación es reflejar el comportamiento de aquellos a quienes deseamos influir o que nos influyan. La imitación de la postura, los gestos, las expresiones faciales e incluso la respiración se puede practicar fácilmente en el aula, mientras que la instrucción simple logra los mismos resultados con las características fonológicas del habla conectada y las frases léxicas clave.

Para lograr una comunicación natural, los aspectos verbales y no verbales deben combinarse en actividades comunicativas. Se puede pedir a los alumnos que reflejen el 
comportamiento de los personajes en la televisión antes de reflejarse entre sí y con el maestro.

De esta manera el mantener el flujo para el PNL encaja muy bien con la 'teoría del flujo', la noción de que el aprendizaje fluye como el agua y que el mejor aprendizaje se produce cuando no se interrumpe. A los efectos de la planificación de lecciones, el flujo se logra cuando existe un equilibrio entre el desarrollo de habilidades y nuevos desafíos, metas claras de tareas y la necesidad de concentración. El aprendizaje exitoso tiene lugar cuando los alumnos tienen una sensación de control sobre lo que está sucediendo en el aula, no se sienten cohibidos y reciben comentarios positivos entre ellos y del maestro.

\section{Metodología}

Sin duda alguna, la pandemia de COVID 19 ha afectado significativamente a la sociedad del conocimiento en el Ecuador; esta investigación utilizó el método cuantitativo con el fin de analizar los hallazgos. Apuke (2017) afirma que «la investigación cuantitativa implica la recolección de datos para que la información pueda ser cuantificada y sometida a un tratamiento estadístico con el fin de apoyar o refutar afirmaciones de conocimiento alternativo». Por lo tanto, el grupo investigador utilizó métodos estadísticos para analizar los resultados iniciales (pre-test) y finales (post-test).

Este estudio es correlacional no experimental. Por esta razón, este tipo de procedimiento se realiza cuando los sujetos a investigación no pueden ser asignados al azar. La investigación correlacional se considera no experimental porque se centra en la relación estadística entre dos variables, pero no incluye la manipulación de una variable independiente. Más específicamente, en la investigación correlacional, el investigador mide dos variables con poco o ningún intento de control de variables extrañas y luego evalúa la relación entre ellas (Jhangiani et al., 1994).

Para el presente estudio de carácter estadístico utilizando método paramétrico como el test estadístico T o T Student para determinar si existe una diferencia significativa en el preprueba y en el post prueba. Garth (2008) indica que los investigadores pueden tener los mismos sujetos para investigar tomando el mismo instrumento en dos momentos diferentes para determinar la estabilidad de sus respuestas. Esto se debe al hecho de que permite identificar una variación principalmente en los medios de los datos obtenidos, lo que permite determinar si el estudio aplicado ha tenido éxito o no. Sin embargo, para este test estadístico se realizó una prueba de validación del instrumento aplicado. La validación de un instrumento permite identificar que los datos tienen la misma distribución y que al aplicar el test los datos pueden ser verificables y reales.

En esta situación se utilizó el test de validación de Shapiro Wilk, el mismo que permite determinar si los datos estudiados corresponden a una distribución normal en la obtención 
de la media aritmética, ya que la muestra estudiada es inferior o igual a 30 individuos. Tarasinska (2016) menciona que el test de Shapiro Wilk trata de rechazar la hipótesis nula a nivel de significación.

Se han considerado por motivos de pandemia tomar a 30 estudiantes, siendo el universo total utilizado para realizar la investigación, la fórmula de muestreo no se realiza por lo que dicha población no requiere por ser mínima. Como menciona Delice (2001), las técnicas de muestreo son un conjunto de técnicas estadísticas que estudian cómo seleccionar una muestra representativa de la población, sobre todo si se trata de grandes cantidades.

Los datos iniciales fueron recolectados mediante una prueba previa para identificar el nivel de los estudiantes en términos de habilidades de habla. Mientras que un post-test se utilizó para mostrar el nivel de los estudiantes al final del proceso. Cabe destacar que para la elaboración de los instrumentos se realizó una operacionalización de variables, lo que corroboró la estructura y eficacia de las evaluaciones y la rúbrica para calificarlas. Estos datos mostraron cómo cambiaron los resultados iniciales al aplicar técnicas de programación neurolingüística, que se reflejan en los resultados finales. Las pruebas pre y post fueron validadas mediante la técnica de validación de contenidos para asegurar que los contenidos inmersos en ellas estén de acuerdo con lo establecido. Para ello, tres profesionales que dictan la catedra de este idioma regularmente en Carreras de Pregrado y Posgrado en Enseñanza de Inglés actuaron como expertos para revisar la relevancia y validez de estos instrumentos. Al igual que en el pre-test y el post-test, todos los profesionales coincidieron en que el test debe incluir 3 partes, la primera inacción maestro-alumno con 4 avisos de respaldo, la segunda interacción estudiante-alumno con 3 avisos de respaldo y finalmente una interacción grupal con 4 avisos de respaldo. La rúbrica de evaluación contendrá 4 criterios de evaluación vocabulario, gramática, comprensión e interacción en los que la puntuación 4 es la más alta y 1 la más baja. Todo esto debido a, determinar el nivel de los estudiantes al principio y al final de la investigación. De acuerdo con lo mencionado, algunas técnicas neurolingüísticas (Anclaje, Crear relación, Espejo y Mantener el Flujo) fueron implementadas durante un periodo de 4 meses a los estudiantes en el horario de inglés, lo cual está incluido en la planificación.

\section{Resultados}

Los datos de la presente investigación se han procesado mediante el Programa Estadístico SPSS Statics utilizando estadística descriptiva y el test $\mathrm{T}$ student para muestras relacionadas. El estudio consistió en la aplicación de un pre prueba y una post prueba a un grupo de 30 estudiantes realizado en la pandemia por COVID 19 en una institución educativa en el Ecuador (PCI Juan Montalvo) específicamente en la asignatura del idioma de inglés. 
La evaluación realizada a cada estudiante consistió en tres partes, cada parte consideró analizar el nivel de inglés en términos de habilidades productivas de los estudiantes. El test se probó mediante una rúbrica compuesta por 4 criterios de evaluación: Vocabulario, Gramática, Comprensión e Interacción. Cada parte fue ponderada con una puntuación máxima de 4 puntos, que, sumada por el número de partes tanto para la preprueba como para la post prueba, da 48 puntos totales respectivamente. Tomando en consideración lo anteriormente expuesto, se analizaron las partes individuales que componen cada prueba para determinar la media y la tendencia estadística individual de cada parámetro de evaluación en los estudiantes. Los gráficos y datos procesados en el programa se detallan a continuación con resultados estadísticos descriptivos:

\section{Figura 1}

Análisis previo y posterior al ensayo

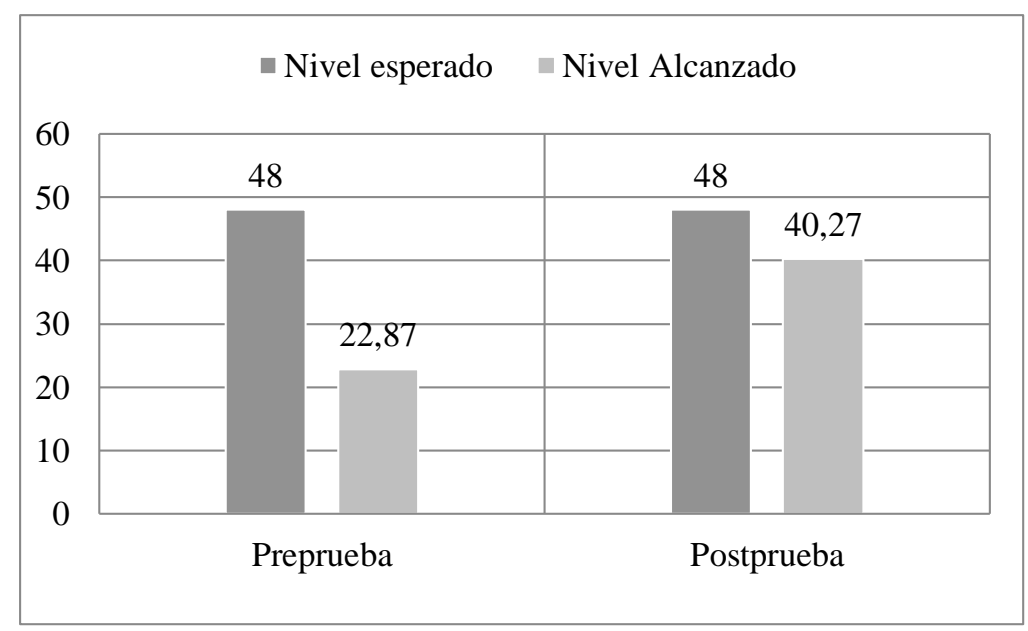

De los 48 puntos esperados en la preprueba, se obtuvo una puntuación media de 22,87. Por otro lado, en el post prueba el puntaje esperado se obtuvo una puntuación de 40,27 en el análisis global. A partir de los datos recogidos en los puntajes globales, se puede identificar que los datos corresponden a una distribución normal y válida aplicando el test estadístico. Los datos verifican que los estudiantes tenían un nivel inferior al 50\% de la puntuación esperada al momento de la preprueba, pero superior al 50\% de la puntuación esperada al momento de la post prueba. Esto se debe a que los estudiantes participaron activamente en cada una de las actividades utilizadas a partir de las técnicas de Programación Neurolingüística, para las cuales se evidencia una mejoría en el desarrollo de habilidades orales y escritas, logrando un estudio factible y verificable.

Como consideración final, puede expresar que, en una comparación restando el 52\% de la media del valor esperado del resultado anterior del $16 \%$ de la media del valor esperado del resultado siguiente, habría una diferencia del $36 \%$. Dando a conocer que se incremente el nivel de conocimiento y el desarrollo de habilidades productivas del idioma inglés antes y después de aplicar las técnicas de Programación Neurolingüística. 


\section{Conclusiones}

- En consecuencia, la educación es un derecho humano innegable que debe estar latente en todo momento y en toda sociedad. La enseñanza y el aprendizaje del idioma inglés en tiempos de pandemia es la piedra angular de sociedades bilingües, igualitarias e inclusivas y uno de los principales motores del desarrollo sostenible en el país, este fortalecimiento de la resiliencia de los sistemas educativos permite a los establecimientos y países responder a los desafíos inmediatos que plantea una reapertura segura de las instituciones en todos sus niveles y los prepara para afrontar de mejor manera en futuras crisis como el COVID 19.

- En este sentido las técnicas de Programación Neurolingüística estudian cómo la comunicación verbal y no verbal afecta a nuestro sistema nervioso y, por lo tanto, aprender a dirigir nuestra mente a través de la comunicación interna con buenas habilidades linguiísticas productivas. Por lo tanto, establecer Técnicas de Programación Neurolingüística como anclaje, espejo y mantener el flujo, nos ayuda a tener un mejor control de nosotros mismos, de nuestras emociones y de nuestras respuestas cuando nos expresamos oralmente.

- El uso de Programación Neurolingüística en el desarrollo de habilidades productivas en inglés en los estudiantes mejoró la capacidad de hablar y escribir, especialmente en los criterios de evaluación asignados (Vocabulario, Gramática, Comprensión e Interacción). Los estudiantes se volvieron más activos y entusiastas en el proceso de enseñanza-aprendizaje. A través de estas técnicas, los estudiantes pudieron expresar su opinión a partir de su propia imaginación, utilizando un mayor número de palabras de vocabulario, ayudando a los estudiantes a producir oraciones con una gramática y estructura correcta. Se puede ver en la puntuación de los estudiantes que la mejoría fue identificada por el desempeño docente-alumno, alumno-alumno y grupal, lo que evidenció una mejor comprensión e interacción entre estas actividades.

- La aplicación de técnicas de programación neurolingüística en el desarrollo de las actividades académicas relacionadas a la enseñanza aprendizaje del idioma inglés durante la pandemia de COVID 19, tuvo un impacto positivo en la mejora de los resultados de aprendizaje y rendimiento de los estudiantes, puesto que estas técnicas tienen la capacidad de desarrollar destrezas productivas de mejor manera, que el uso de métodos convencionales o tradicionales. La PNL tienen la capacidad de absorber los problemas que enfrentan, hacer que los estudiantes se sientan seguros, especialmente cuando hablan, aumentar la motivación para hablar y escribir mejorando el rendimiento con pensamientos que recorren sus propios cerebros sin ningún estímulo.

- Después de la implementación de Programación Neurolingüística ante la crisis 
suscitada por COVID 19 en Ecuador, esto hace que se aproxime al desarrollo de habilidades productivas en los estudiantes donde las clases fueron más activas. Se observó durante el proceso de enseñanza-aprendizaje, cuando el investigador hacía preguntas o asignaciones en grupos, que la mayoría de los estudiantes interactuaban entre sí, algunos de ellos intentaban responder a las preguntas, aunque el investigador no les pedía que las respondieran. Antes de la implementación de la Programación Neurolingüística se observaba poca participación de los estudiantes y clases monótonas y pasivas; no había interacción entre los alumnos y apenas participaban en las actividades del aula.

- Finalmente, las técnicas de programación neurolingüística dio resultados positivos en los estudiantes pese a la crisis por COVID 19, se observó la aproximación que existió para lograr el desarrollo de habilidades productivas y lograr con mayor eficiencia la enseñanza del idioma inglés.

\section{Referencias bibliográficas}

Apuke, O. D. (2017). Quantitative Research Methods: A Synopsis Approach. Kuwait Chapter of Arabian Journal of Business and Management Review, 6(11), 40-47. https://doi.org/10.12816/0040336

Arlinthon, D. \& Pomares, M. (diciembre de 2011). Programación neurolinguiística. ¿Realidad o mito en Psicología y Ciencias Cognitivas? Duazary, 246. https://www.redalyc.org/pdf/5121/512156315015.pdf

Bandler, R. \& Gindler, J. (1980). La estructura de la magia. Cuatro Vientos.

Castro, S. (2020). Instituto Europeo de Psicología Positiva. https://www.iepp.es/comunicacion-efectiva/

Delice, A., (2001). "The Sampling Issues in Quantitative Research", Educational Sciences: Theory and Practice, 10(4), 2001-2018, 2010.

Estupiñan-Ricardo, J. (2017). La influencia de la programación neurolingüística en estudiantes universitarios en la. Luz, https://www.redalyc.org/pdf/5891/589166502012.pdf

Garth, A. (2008). Analysing data using SPSS (A practical guide for those unfortunate enough to have to actually do it.). 
Jhangiani, R., Chiang, I., Cutteler, C., \& Leighton, T. (1994). Research Methods in Psychology. Journal of Environmental Psychology, 14(4), 335. https://doi.org/10.1016/s0272-4944(05)80228-2

Proaño, C., Pila, J. \& Venegas, G. (2017). Potencialización de la competencia comunicativa en inglés a través de pares receptivos y productivos. Scientific Report 1st English Teaching Congress (p. 101). CIDE. http://repositorio.cidecuador.org/bitstream/123456789/40/1/Scientific\%20Report $\% 201$ st\%20English\%20Teaching\%20Congress.pdf

Tarasinska, J. (2016). Shapiro - Wilk Test with Known Mean. 14(1), 89-100.

Trujillo-Sáez, F., Fernández-Navas, M., Montes-Rodríguez, M., Segura-Robles, A., Alaminos-Romero, F.J. \& Postigo-Fuentes, A.Y. (2020). Panorama de la educación en España tras la pandemia de COVID-19: la opinión de la comunidad educativa. Madrid: $\quad$ Fad. $\quad$ DOI: $\quad 10.5281 /$ zenodo-3878844. https://digibug.ugr.es/bitstream/handle/10481/62687/COVID-INFORME2.pdf?sequence $=1 \&$ is Allowed $=\mathrm{y}$

Vargas, V. (2021). tesis.unap.edu.pe. Obtenido de La comunicación efectiva como estrategia didáctica en las competencias lingüísticas para el aprendizaje del idioma inglés como tercera lengua en estudiantes andinos.: http://tesis.unap.edu.pe/bitstream/handle/UNAP/16828/Vargas_Velasquez_Diana _Agueda.pdf?sequence $=1 \&$ isAllowed $=y$

Yameen, A., \& Iftikhar, L. (2014). Neurolinguistic Programming as an Instructional Strategy to Enhance Communicative Competence of Language Teachers. Journal of Applied Environmental and Biological Sciences, 4, 331-336. https://www.textroad.com/pdf/JAEBS/J. Appl. Environ. Biol. Sci., 4(7S)331-336, 2014.pdf

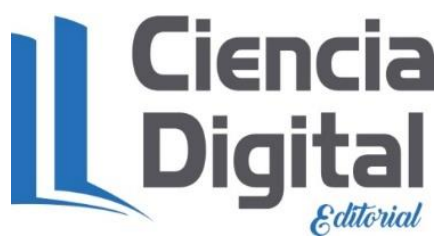


El artículo que se publica es de exclusiva responsabilidad de los autores y no necesariamente reflejan el pensamiento de la Revista Conciencia Digital.

\section{Ciencia}

El artículo queda en propiedad de la revista y, por tanto, su publicación parcial y/o total en otro medio tiene que ser autorizado por el director de la Revista Conciencia Digital.
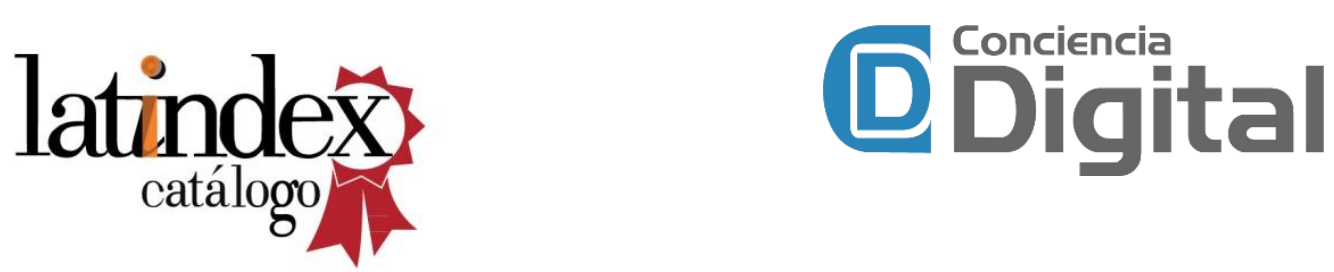

Indexaciones

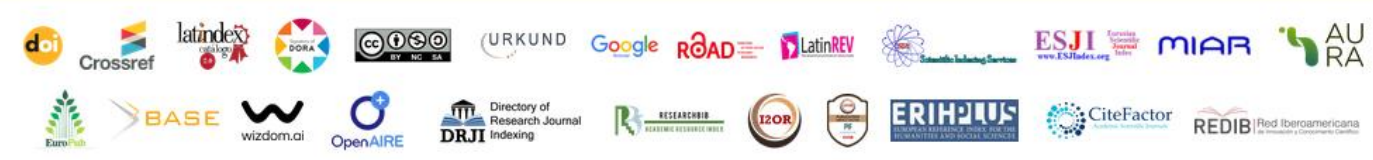

